\title{
Neruda, poète ou malacologue
}

Neruda, poet or malacologist

Sara Contreras et Michel Étienne

\section{OpenEdition}

Journals

Édition électronique

URL : https://journals.openedition.org/tc/6781

DOI : $10.4000 /$ tc. 6781

ISSN : 1952-420X

\section{Éditeur}

Éditions de l'EHESS

\section{Édition imprimée}

Date de publication : 15 décembre 2012

Pagination : 346-357

ISBN : 978-2-7351-1534-

ISSN : 0248-6016

\section{Référence électronique}

Sara Contreras et Michel Étienne, « Neruda, poète ou malacologue », Techniques \& Culture [En ligne], 59 | 2012, mis en ligne le 29 juillet 2013, consulté le 29 septembre 2022. URL : http:// journals.openedition.org/tc/6781; DOI : https://doi.org/10.4000/tc.6781 

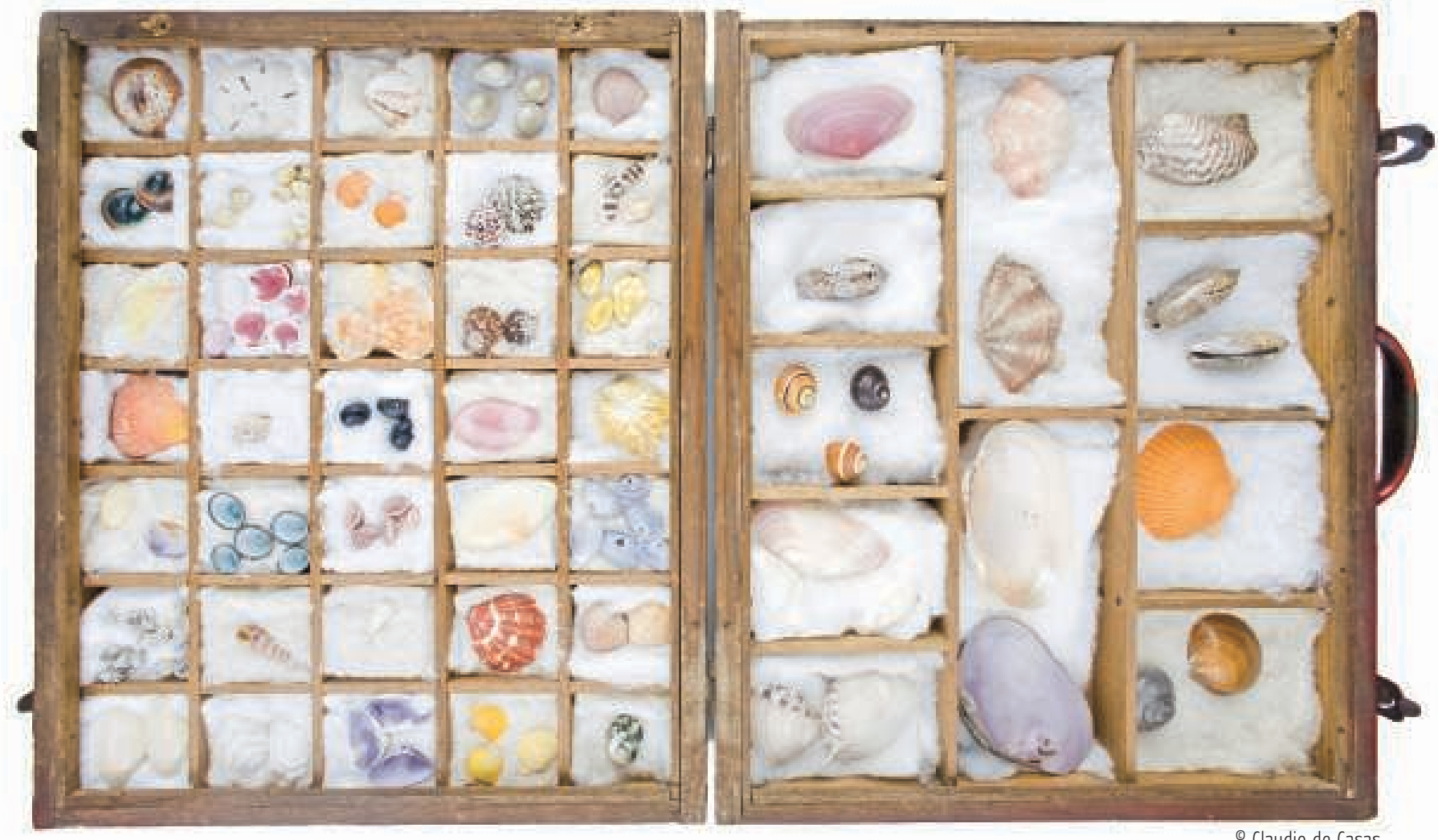

- Claudio de Casas 


\section{Sara Contreras \& Michel Étienne}

INRA

etienne@avignon.inra.fr

Techniques E Culture 59, 2012/2: 346-357

\section{NERUDA, POĖTE OU MALACOLOGUE}

Une anecdote couramment colportée au Chili du temps de la dictature rapporte que le biologiste Julian Huxley (le frère d'Aldous) à son arrivée à l'aéroport de Santiago du Chili ${ }^{1}$ avait dit aux journalistes qu'il souhaitait rencontrer Pablo Neruda. À l'interrogation des journalistes : «Pablo Neruda le poète? », il avait répondu : « Non, non, Pablo Neruda le malacologue».

Pablo Neruda raconte dans sa biographie posthume Confieso que he vivido (J'avoue que j'ai vécu) que « l'historiette destinée à me vexer, me fit plaisir, d'autant plus qu'elle ne pouvait pas être vraie puisque nous nous connaissions, Huxley et moi, depuis longtemps [1] ». Il raconte alors comment « Au Mexique, [il] parti[t] explorer les plages, plongeant dans les eaux transparentes et chaudes, et ramassant de merveilleux coquillages » [1].

Le hobby d'habitant du bord de mer va se transformer, après sa rencontre avec le grand malacologue cubain Carlos De La Torre qui lui offre «les plus jolies pièces de sa collection » [1], en une fascination pour les formes et les couleurs de ces créatures extraordinaires: « littorines antarctiques et hélix cubains, ou vigneaux-peintres vêtus de rouge et de safran, de bleu et de violet, comme des danseuses des Caraïbes » [1]. Puis elle va devenir une passion dévorante.

« Par la suite, à Cuba et ailleurs, mais aussi par des échanges et des achats, des cadeaux et des vols (il n'existe pas de collectionneur honnête), mon trésor marin s'accrut au point de remplir des pièces et des pièces de ma maison. Je me mis à posséder les espèces les plus rares des mers de Chine et des Philippines, du Japon et de la Baltique » [1].

Il invente d'ailleurs un mot pour définir cette passion - caracolismo - et un verbe pour sa transmission à ses amis - encaracolarse.
En réalité, ma plus belle collection aété constituée par les coquillages. Ils m’ont donné le plaisir de leur structure prodigieuse, la pureté lunaire d'une porcelaine mystérieuse alliée à la multiplicité de leurs formes, tactiles, gothiques, fonctionnelles.

Pablo Neruda 


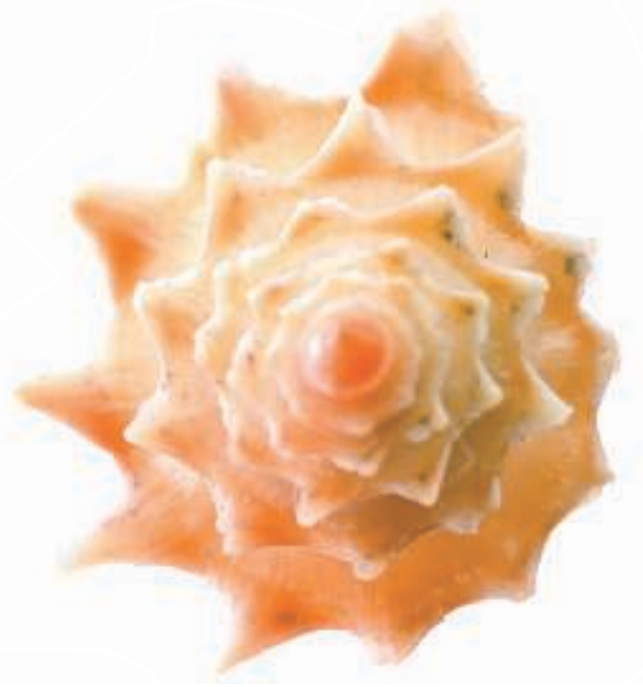

Colombarium pagoda
« Mon coquillagisme me poussa à visiter des mers lointaines. Mes amis, à leur tour, se mirent à chercher des littorines, à s'encoquillager » [1].

Mettant à profit son aura internationale ou son statut de représentant plénipotentiaire du Chili, il se fit offrir des exemplaires rarissimes.

« Je me souviens qu'au musée de Pékin on ouvrit la caisse la plus sacrée des mollusques de la Mer de Chine pour m'offrir le second des deux seuls spécimens de la Thatcheria mirabilis. Je pus ainsi enrichir ma collection d'un exemplaire dans lequel l'océan avait fait don à la Chine d'un style qui a survécu : celui de ses temples et de ses pagodes » [1].

Son admiration devant ces spécimens exceptionnels est démultipliée par sa capacité à les décrire et à s'en servir pour stimuler son imagination poétique ou sa prose féconde. Il va ainsi raconter ses trouvailles au marché aux puces de Paris:

«Apercevoir sous les sargasses de la capitale, entre des lampes démantibulées et de vieux souliers, l'exquise silhouette de l'Olivia textil, ou surprendre la lance de quartz qui s'allonge, comme un verset d'eau, dans la Rostellaria fusus » [1].

Il va aussi faire part de son éblouissement quand lors de fouilles marines:

« J'arrachai à la mer l'Espondylus rose, une grande huître sertie d'épines de corail... quand j'entrouvris l'Espondylus blanc, aux piquants neigeux comme des stalagmites $»^{2}[1]$.

Il va surtout parsemer ses poésies de références nombreuses à ces « petites portes sous-marines » [1]. Elles y jouent des rôles multiples soit pour ce qu'elles sont (la carapace d'un mollusque), soit pour ce qu'elles pourraient représenter dans l'imaginaire du poète ou des peuples qui les ont inscrites dans leur patrimoine symbolique.

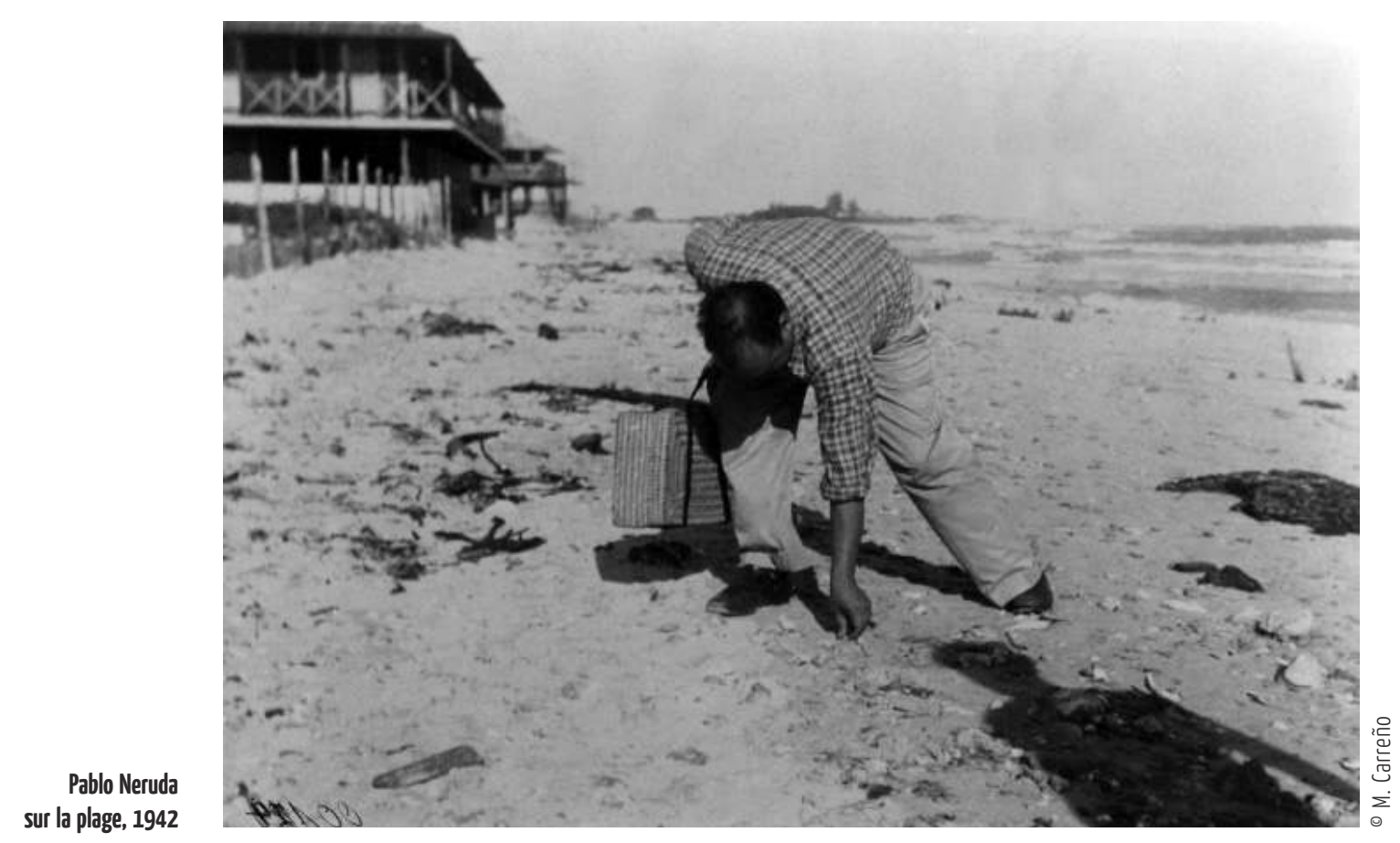


Les coquillages sont alors sirène d'alarme et cor de ralliement guerrier pour soutenir les révoltes indigènes:

"Tiraron la manta en el polvo,

se unieron los viejos cuchillos,

y la caracola marina

llamò los vínculos dispersos.

Contra la piedra sanguinaria,

contra la inercia desdichada,

contra el metal de las cadenas. »

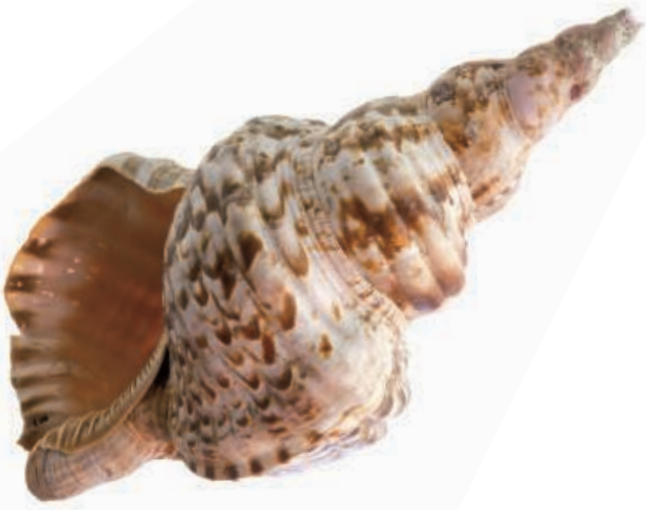

« Tupac Amaru », Canto General

ou prétexte à un hommage aux scaphandriers qui risquent leur vie pour ramener quelque pitance des entrailles de la mer.

«Vuelven con un puñado de palpitantes frutos submarinos, gòticas caracolas, erizados erizos:

el buzo emerge de la mitología en su escafandra, pudo bailar con las medusas,

quedarse en el profundo hotel de las sirenas. »

«Oda al espacio marino », Nuevas odas elementales

Ils sont aussi interprétés comme des symboles de féminité, voire d'érotisme,

« Son los senos de las sirenas

las redondescas caracolas?"

Libro de las preguntas

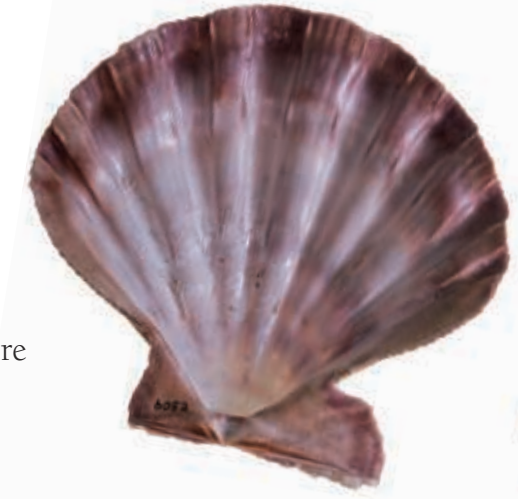

Pecten nodosus
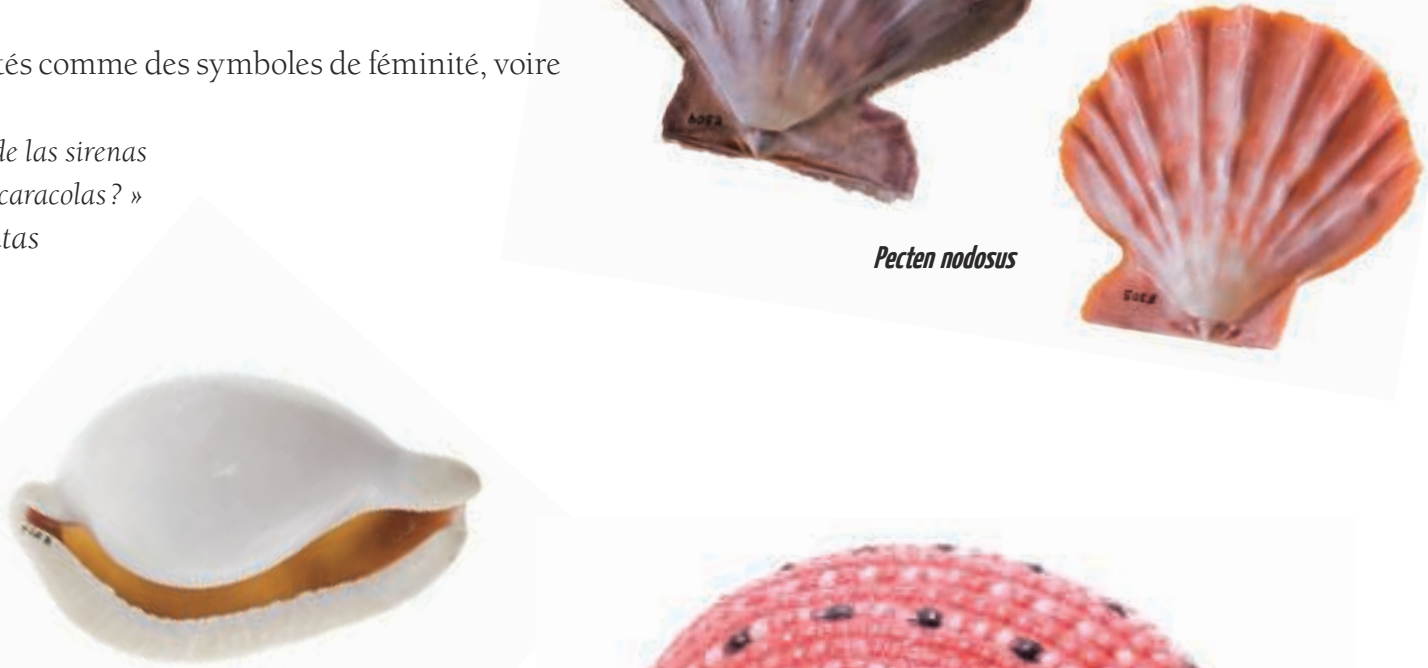

Ovula ovum

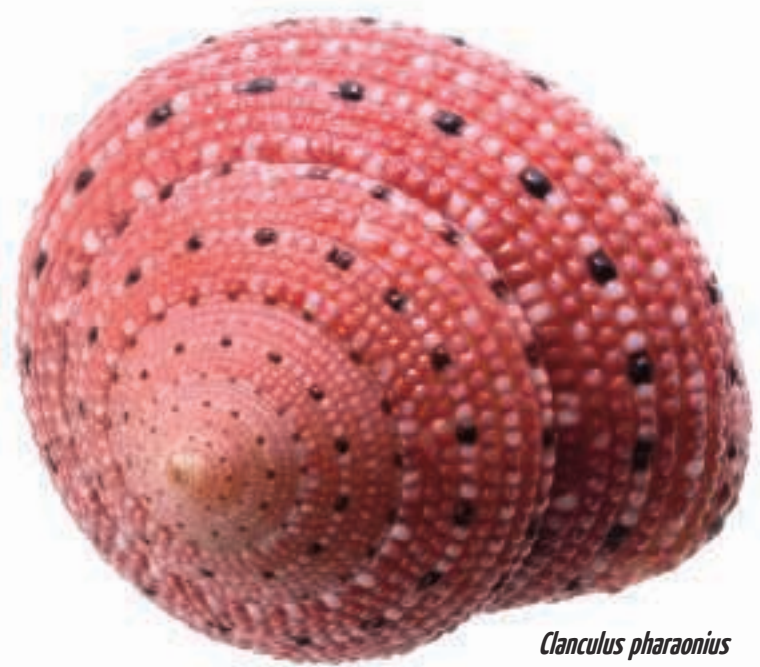




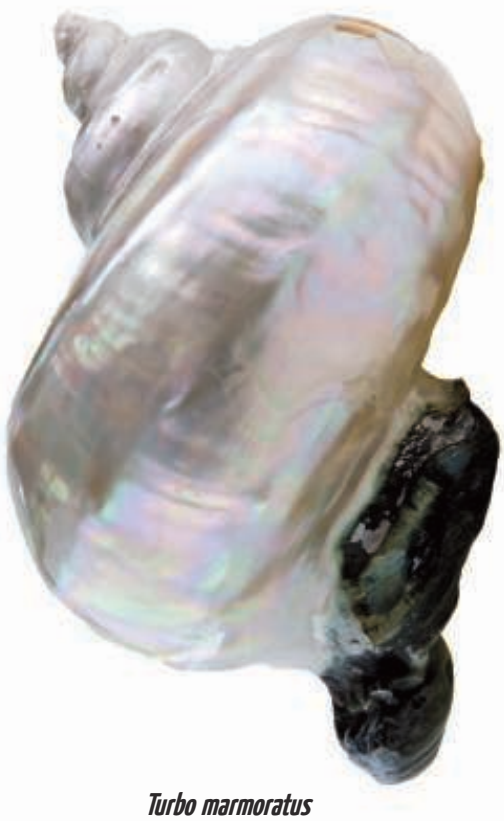

Murex pecten

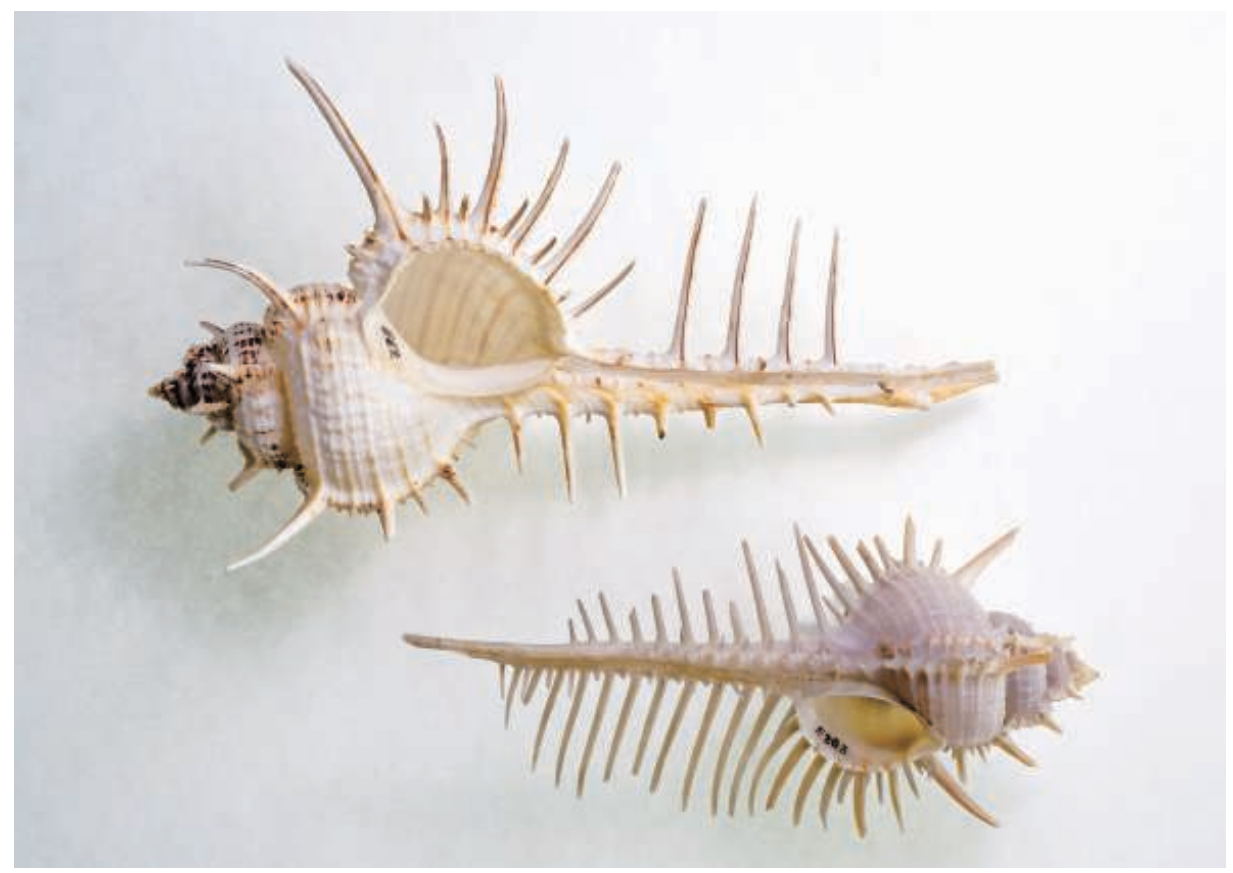

ou l'enregistrement secret d'une chanson magique qui ouvre les portes du temps «Sí, pero tengo tiempo,

tengo aún mucho tiempo

-tengo una caracola que recoge

la tenaz melodía del secreto

y la guarda en su caja

convertida en martillo o mariposa »

«Odas de todo el mundo », Odas elementales II

ou une sorte de fin métaphysique difficile à atteindre.

«Ay cuánta noche cabe en una noche

sin desbordar esta celeste copa,

suena el silencio de las lejanías

como una inaccesible caracola

y caen en mis manos las estrellas

llenas aún de música y de sombra. "

« Escrito En El Año 2000 », Cancion de gesta

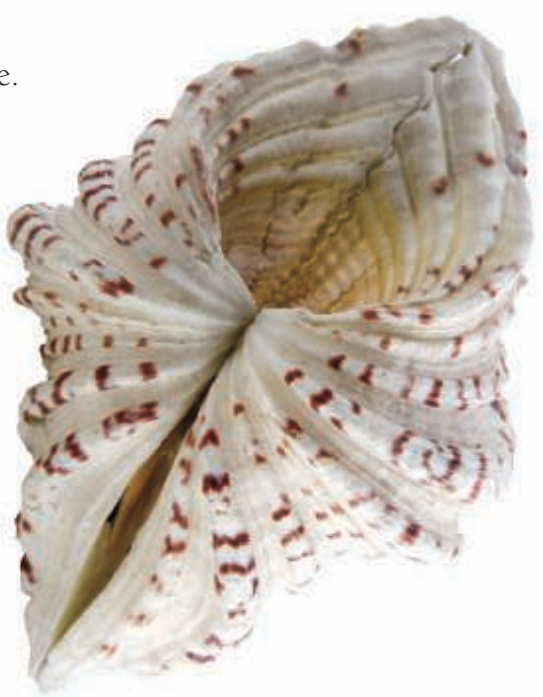

Hippopus hippopus

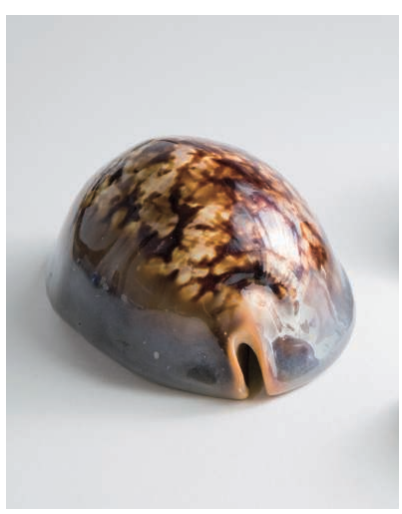




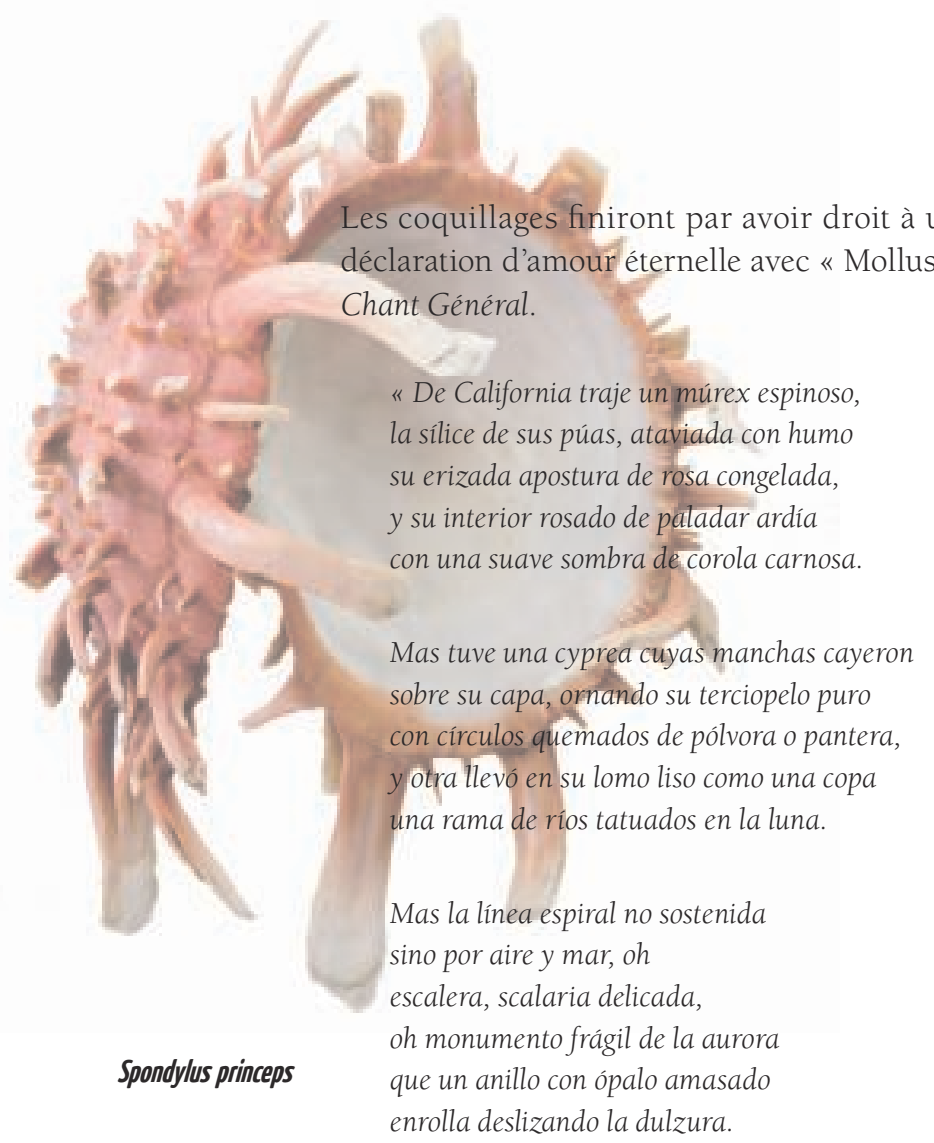

Saqué del mar, abriendo las arenas, la ostra erizada de coral sangriento, spondylus, cerrando en sus mitades la luz de su tesoro sumergido, cofre envuelto en agujas escarlatas, o nieve con espinas agresoras.

La oliva grácil recogí en la arena, húmeda caminante, pie de púrpura, alhaja humedecida en cuya forma la fruta endureció su llamarada, pulió el cristal su condición marina y ovaló la paloma su desnudo.

La caracola del tritón retuvo la distancia en la gruta del sonido y en la estructura de su cal trenzada sostiene el mar con pétalos, su cúpula.

Oh rostellaria, flor impenetrable como un signo elevado en una aguja, minima catedral, lanza rosada, espada de la luz, pistilo de agua.
J'apportai de Californie un murex épineux, la silice dans ses piquants; la fumée habillait sa prestance hérissée de rose congelée, et dans son intérieur une muqueuse ardente de palais flambait avec une ombre douce de corolle charnue.

Et je trouvais une cypris dont les taches tombaient sur sa cape et ornaient son velours pur de cercles calcinés de poudre ou de panthère.

Une autre portait sur son dos lisse comme une coupe une branche de fleuves tatoués sur la lune.

Et je trouvai aussi la spirale que seuls l'air et la mer soutenaient, ô échelle, scalaire délicate, ô monument fragile de l'aurore qu'un anneau pétri dans l'opale enroule en glissant la douceur.

J'ai sorti de la mer, en écartant le sable, l'huître épineuse de corail sanglant, le spondyle enserrant dans ses moitiés la clarté de son trésor submergé, coffre enveloppé d'aiguilles écarlates ou neige aux piquants agressifs

J'ai trouvé l'olive gracile sur le sable, marcheuse mouillée, pied de pourpre, humide bijou, forme dans laquelle le fruit a durci sa rouge flambée, le cristal, poli sa condition marine, et la colombe façonné l'ovale de son nu.

La conque du triton a retenu la distance dans la grotte du son et sur sa structure de chaux tressé la mer soutient sa coupole, avec des pétales.

Ô rostellaire, fleur impénétrable comme un signe élevé sur une aiguille, minime cathédrale, lance rose, épée de la clarté, pistil de l'eau. 

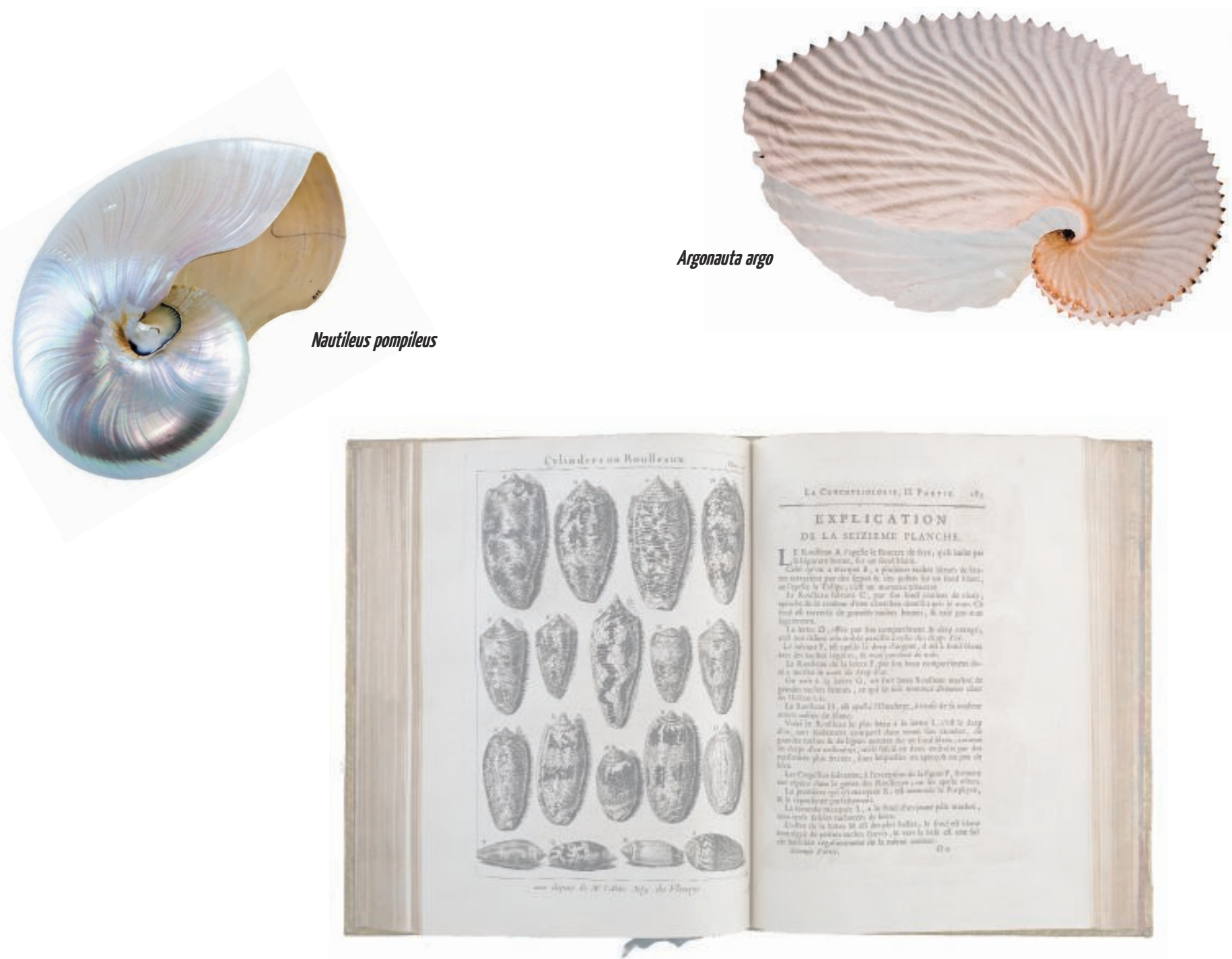

En 1954, sa collection avoisine les 15000 exemplaires et devient tellement envahissante qu'il décide de s'en libérer, avec les ouvrages scientifiques associés qui emplissent sa bibliothèque. Il choisit alors d'en faire don à l'Université du Chili.

Le discours qu'il prononce à cette occasion montre l'importance et la forte valeur symbolique quavait pour lui cette collection.

«J'ai recueilli ces livres de la culture universelle, ces coquillages de tous les océans et cette écume des sept océans je la donne à l'Université, par devoir de conscience et pour payer une part infime de ce que j'ai reçu de mon peuple La splendeur de ces livres, la gloire océanique de ces coquillages, tout ce que j'ai accumulé tout au long de ma vie, malgré la pauvreté et par un travail constant, je le remets à l'Université, c'est-à-dire, je le donne à tous » [2].

La façon dont il raconte cet épisode de sa vie dans ses mémoires posthumes laisse apparaître une profonde blessure:

«Ma collection avait sa célébrité. En bonne institution sud-américaine, mon université la reçut avec des louanges et des discours et l'enterra dans un sous-sol. On ne l'a plus revue depuis » [1]. 


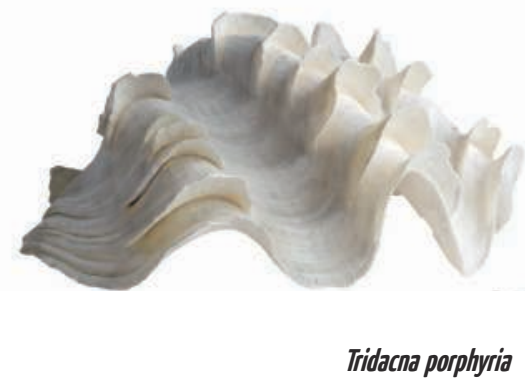

iridacna porphyria

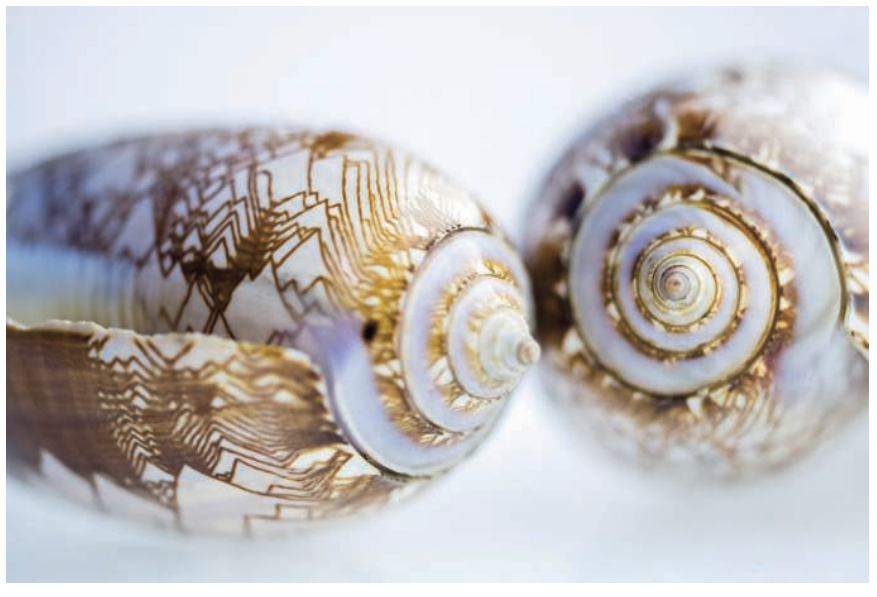

Oliva porphyria

Ce n'est que trente-cinq ans après sa mort et cinquante-cinq ans après sa donation que l'outrage sera réparé, lors de l'organisation à Madrid, par l'Institut Cervantes, l'Ambassade du Chili en Espagne et l'Université du Chili de l'exposition « Amor al mar. Las caracolas de Neruda » (Amour de la mer. Les coquillages de Neruda).

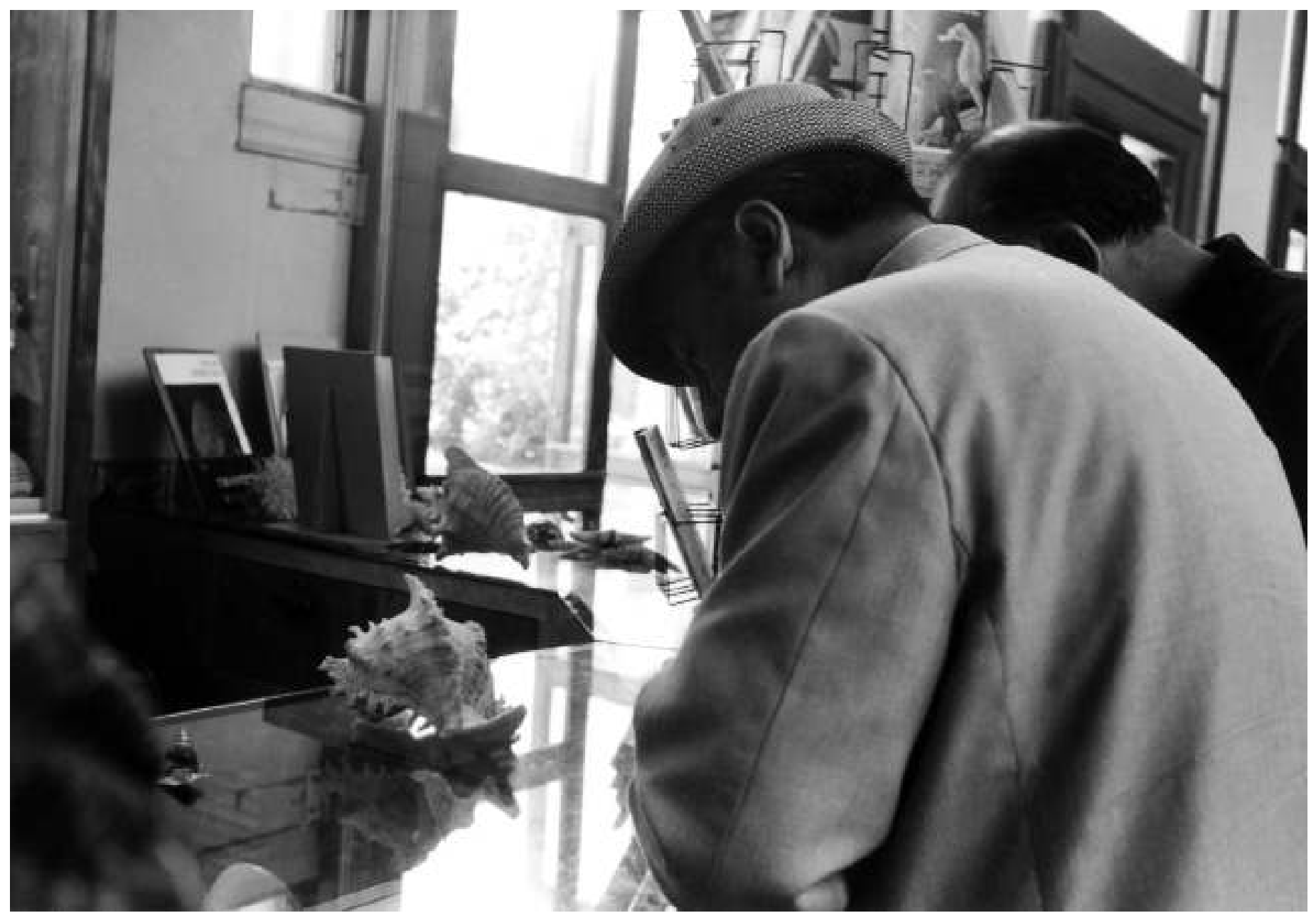




\section{NOTES}

Photo d'ouverture: Material de coleccionista (antiques) / Matériel de collectionneurs (Antiquités). Toutes les photographies sont de Claudio de Casas (Exposition « Amor al mar. Las caracolas de Neruda », Institut Cervantes), à l'exception des deux portraits en noir et blanc respectivement de Mario Carreño et Matilde Urrutia.

- $\quad$ Les textes entre guillemets suivis d'un [1] sont extraits de la traduction française par Claude Couffon de « Confieso que he vivido » (Gallimard). Les textes marqués d'un [2] sont extraits de la traduction du discours de Pablo Neruda à l'occasion de la donation de sa bibliothèque et de sa collection de coquillages à l'Université du Chili, en 1954.

- Les photographies de coquillages sont toutes, sauf celle du nautile, extraites du catalogue de l'exposition « Amor al mar. Las caracolas de Neruda » au crédit de Claudio de Casas.

- Les photographies en noir et blanc sont de Mario Carreño (La plage, 1942) et de Matilde Urrutia (Le magasin de coquillages, 1966).

1. Dans son livre Neruda, Volodia Teitelboim, situe l'anecdote dans la maison du poète à Michoacan au Mexique!
2. La façon espagnole ou latino-américaine de prononcer le «sp » dans toutes les autres langues du monde fait de Spondylus princeps, l'Espondylus róseo et de Spondylus imperialis l'Espondylus blanco.

\section{REMERCIEMENTS}

La rédaction de Techniques E Culture tient à remercier très chaleureusement les auteurs et détenteurs des œuvres et droits cédés gracieusement pour la publication de cet article : Matilde Urrutia et Mario Carreño, et tout particulièrement Claudio de Casas, Pedro Núñez commissaire de l'exposition « Amor al mar. Las caracolas de Neruda » pour l'Institut Cervantes ainsi que Fernando Sáez, Javier Ormeño et Ana Paz pour la Fondation Pablo Neruda, enfin Kepa Gonzales Lopez, directeur culturel de l'Institut Cervantes de Manchester pour son aide précieuse. 


\section{RÉFÉRENCES}

Neruda, P. 1950 Canto General. Mexico: Talleres Gráficos de la Nación.

- 1954 Odas elementales. Buenos Aires: Losada.

- 1954 Discurso en la donación de su biblioteca personal a la Universidad de Chile, el 20 de junio de 1954

- 1956 Nuevas odas elementales. Buenos Aires: Losada.

- 1960 Canción de gesta. La Habana: Imprenta Nacional de Cuba.

— 1974 Libro de las preguntas. Buenos Aires: Losada.

- 1974 Elegía. Buenos Aires: Losada, 1974.

— [1974] 1975 Confieso que he vivido. Paris: Gallimard.

- 2010 Canto General. Traduction Claude Couffon. Paris: Gallimard (NRF, Poésie) : 468-470

Teitelboim, V. 1984 Neruda.

\section{Expographie}

Amor al mar. Las caracolas de Neruda. Madrid: Institut Cervantes 2009.

\section{MOTS-CLÉS}

Neruda, coquillages, Canto General, malacologie, poésie, Chili

\section{KEYWORDS}

Neruda, shells, Canto General, malacology, poetry, Chile

\section{PALABRAS CLAVE}

Neruda, caracolas, Canto General, malacología, poesía, Chile 